\title{
Magnetic observatories: biases over CHAMP satellite mission
}

\author{
G. Verbanac ${ }^{1}$, M. Mandea ${ }^{2}$, M. Bandić ${ }^{3}$, and S. Subašić ${ }^{4}$ \\ ${ }^{1}$ Department of Geophysics, University of Zagreb, Horvatovac 95, Zagreb, Croatia \\ ${ }^{2}$ Centre National d'Etudes Spatiales 2 Place Maurice Quentin, 75039 Paris, France \\ ${ }^{3}$ Preziosastr. 15a, 81927 Munich, Germany \\ ${ }^{4}$ Vukomerec 41, 10000 Zagreb, Croatia
}

Correspondence to: G. Verbanac (giuli1.verbanac@gmail.com)

Received: 03 October 2014 - Published in Solid Earth Discuss.: 30 January 2015

Revised: 28 April 2015 - Accepted: 05 May 2015 - Published: 26 June 2015

\begin{abstract}
Taking advantage of 9 years of the CHAMP (CHAllenging Minisatellite Payload) satellite mission (June 2000-August 2009), we investigate the temporal evolution of the observatory monthly magnetic biases. To determine these biases we compute $X$ (northward), $Y$ (eastward) and $Z$ (vertically downward) monthly means from 42 observatory $1 \mathrm{~min}$ values or hourly values, and compare them to synthetic monthly means obtained from a $\mathrm{G}$ field model (Lesur et al., 2015). Afterwards, the average of biases at all observatories over 9 years is calculated and analyzed. Both the long-term trends and short-period variations (hereafter $\epsilon$ variations) around these averages are then investigated. The simple oscillatory pattern of $\boldsymbol{\epsilon}$, found at all observatories and in each component over the entire considered period, indicates that the crustal field has not changed. A comparison with both MAGSAT and Ørsted biases given for epochs 1979.92 and 1999.92 which are based on 2 single months (November and December) of MAGSAT and Ørsted satellite data, respectively, further shows that the crustal field has probably remained constant over last 3 decades. The long-trend seen in $\epsilon$ reflects the changes within the solar cycle 23 . The short period variations observed in the $\epsilon$ time series are related to the external field. The amplitudes of these variations are found to be in phase with solar cycle periods, being remarkably larger over 2000-2005 than 2006-2009. Furthermore, clear semi-annual variations are observed in $\boldsymbol{\epsilon}$, with larger extremes appearing mostly around October and November, and around May and June of each year in $X$, and vice versa in $Y$ and $Z$. A common external field pattern is found for the European monthly biases. The dependence of the bias monthly variations on geomagnetic latitudes is not found for non-European observatories. The
\end{abstract}

results from this study represent a base to further exploit the magnetic biases computed for observatories and repeat station locations together by using data from the new satellite mission Swarm.

\section{Introduction}

Measured at any point on Earth's surface the magnetic field is a combination of several magnetic contributions generated by various sources. These fields are superimposed on and interact with each other. A dominant part of Earth's magnetic field, the core field (Jacobs, 1987; Merrill et al., 1998), is internal in origin and is due to the electric currents in Earth's outer fluid core. This part of the field varies over timescales of some months to decades and longer. The crustal field, related to the remanent and induced magnetization of rocks within the crust (e.g., Mandea and Thèbault, 2007), is also internal in origin. The magnitude of the crustal field varies from fractions of nanoteslas (nT) to hundreds of nanoteslas but can reach values as high as several thousands of nanoteslas. The external fields are produced by ionospheric and magnetospheric current systems (e.g., Campbell, 2001). The values of those fields at Earth's surface are of a few tens of nanoteslas and even of a few hundred to thousands of nanoteslas during magnetic storms. The external fields vary in time with periods from less than a second to the well-known solar cycle ( $\sim 11$ years) and its harmonics. For an accurate determination of the core field and its temporal variation, it is essential to use geomagnetic observatory data, reprocessed for reflecting as well as possible the core contribution. Thus, contributions of other sources have to be determined and taken into 
account. Note that omitting the possible crustal field contributions may already lead to errors of about $10 \%$ of the field for the large scales (Langel and Hinze, 1998).

Over the last years two different methods have been used to determine the observatory biases. One is to estimate the biases directly as additional unknowns during the fitting process when inverting observatory and satellite data for a spherical harmonic model (Langel et al., 1982; Sabaka et al., 2002). The other method is to compare the magnetic components measured at the observatory to values predicted by a model obtained from satellite data only (e.g., Gubbins and Bloxham, 1985; Bloxham and Gubbins, 1986). Most of the published biases are related to specific epochs, being computed from 1 or several months of satellite data (Mandea and Langlais (2002) and the references therein). Nevertheless, some studies also investigated the temporal changes of the observatory biases over longer periods, by comparing the observatory annual means with models that cover different epochs (Verbanac et al., 2007a).

In this study we analyze monthly observatory bias evolutions of the $X$ (northward), $Y$ (eastward) and $Z$ (vertically downward) magnetic components at 42 observatories. We focus on any temporal changes in the biases and examine their evolution over several years to 3 decades (long-term trends) as well as their variations on a timescale of a several months to 1 year (short period variations). We consider that the longterm trends (changes in the crustal magnetization or induction effects in the lithospheric conductivity anomalies) are likely internal in origin, while the short term variations are related to the external field contribution. The influences of the different phases of the solar cycle 23 on the observatory biases are also examined.

The paper is organized as follows. After this introduction, Sect. 2 describes the theoretical framework of this study and the considered approach to analyze different signals contained in the monthly observatory biases over the 9 years of the CHAMP (CHAllenging Minisatellite Payload) satellite mission (June 2000-August 2009). These monthly observatory biases are calculated by comparing the observatory data to the core field predictions given by the G model (Lesur et al., 2015). In Sect. 3 the used data sets are described. Afterward, we present the obtained results and a comparison with biases based on 2 months of MAGSAT and Ørsted satellite data, related to the 1979.92 and 1992.92 epochs. Conclusions are given in the last section.

\section{Method}

We consider that the geomagnetic field at a given time and observatory location, $\boldsymbol{B}_{\text {obs }}$, can be represented as the vector sum:

$\boldsymbol{B}_{\mathrm{obs}}=\boldsymbol{B}_{\mathrm{m}}+\boldsymbol{B}_{\mathrm{c}, \text { rem }}+\boldsymbol{B}_{\mathrm{c}, \text { ind }}+\boldsymbol{B}_{\mathrm{e}}+\boldsymbol{B}_{\mathrm{err}}$,

where $\boldsymbol{B}_{\mathrm{m}}$ is the main (core) field, $\boldsymbol{B}_{\mathrm{c}, \text { rem }}$ is the remanent crustal field, $\boldsymbol{B}_{\mathrm{c}, \text { ind }}$ is the induced crustal field of both in- ternal and external origin, $\boldsymbol{B}_{\mathrm{e}}$ is the external field and $\boldsymbol{B}_{\text {err }}$ are other contributions, which contain any kind of possible contribution and data errors.

Since it is difficult to make a separation between the remanent and the induced crustal field (Lesur and Gubbins, 2000) we refer to the sum $\boldsymbol{B}_{\mathrm{c}, \text { rem }}+\boldsymbol{B}_{\mathrm{c} \text {, ind }}$ as the crustal field, $\boldsymbol{B}_{\mathrm{c}}$.

Now, we define the observatory magnetic bias $\boldsymbol{B}_{\text {bias }}$ as

$\boldsymbol{B}_{\text {bias }}=\boldsymbol{B}_{\mathrm{c}}+\boldsymbol{\epsilon}$,

where $\boldsymbol{\epsilon}$ contains influences from the external field and from errors in the input data $\left(\boldsymbol{\epsilon}=\boldsymbol{B}_{\mathrm{e}}+\boldsymbol{B}_{\text {err }}\right)$.

With the assumption that in the geomagnetic core models obtained from satellite data the effects of both external and crustal fields are minimized as much as possible, we compute the observatory biases ( $\left.X_{\text {bias }}, Y_{\text {bias }}, Z_{\text {bias }}\right)$ as differences between the magnetic components measured at a given observatory $\left(X_{\mathrm{obs}}, Y_{\mathrm{obs}}, Z_{\mathrm{obs}}\right)$ and the same components calculated at the observatory location from a satellite-based model $\left(X_{\mathrm{m}}, Y_{\mathrm{m}}, Z_{\mathrm{m}}\right)$. The analyzed magnetic field components are given in geodetic coordinates and the described method is applied to monthly means of all three components.

To examine the temporal variability of both bias terms as given in Eq. (2) we apply the following steps. We first compute the average of the bias for each observatory over the studied period (2000-2009) and analyze the temporal variations around the average bias. Two different types of temporal variations around the average bias are possible: (1) simple oscillatory pattern and (2) long-term changes around the average bias. The first kind of variation indicates that $\boldsymbol{B}_{\mathrm{c}}$ does not change within the studied time span. In this case, the average of bias is a good measure of the crustal field over 9 years studied and the variation around average bias corresponds to $\epsilon$. The second type of variation suggests that $\boldsymbol{B}_{\mathrm{c}}$ has indeed changed over the considered time. Now, the sum of the average bias and long-term trend in the variation around average bias would be a representative measure of the crustal field. Then, the remaining variations around the crustal field corresponds to $\epsilon$. After performing this analysis, we further examine the long-term trend in $\epsilon$ related to the solar cycle phases, as well as the remaining short-period variations in $\epsilon$ connected to the external field.

\section{Data sets}

One data set consists of the monthly means computed from the available continued measurements at magnetic observatories, from June 2000 to August 2009. For all 42 geomagnetic observatories, the monthly means are calculated from 1 min values or hourly means when the 1 min values are not available.

The initial data sets were obtained from INTERMAGNET (http://www.intermagnet.org) and WDC Edinburgh (http:// www.wdc.bgs.ac.uk). We have checked the availability, quality and continuity of the observatory data over the studied pe- 
riod. Monthly means have been calculated only if more than $90 \%$ of the $1 \mathrm{~min}$ or hourly values are available, ensuring the reliability of our final monthly means data set. All changes in the observatory locations and data discontinuities (jumps) reported in their annual mean values are taken into account by adjusting the geomagnetic measurements to the level of the most recent epoch. The correction values obtained from the British Geological Survey (http://www.geomag.bgs. ac.uk/data_service/data/annual_means.shtml) have been applied to the following observatories: EBR, ESK, HLP, LER TRO, WNG, NVS, and PPT.

The second data set is based on synthetic monthly means estimated for the same observatory locations and for the same time span as above from a magnetic field model, here $\mathrm{G}$ model. This model is entirely based on data provided by the CHAMP satellite. We have used the internal field descriptions up to the spherical harmonic degree and order of 14 .

\section{Results}

In the following we present the results on the monthly biases temporal evolution, the biases estimated as described in Sect. 2.

\subsection{Average observatory biases}

We first compute the average of the bias for each observatory over 9 years (June 2000-August 2009) at different locations. The averaged biases for all considered observatories with the corresponding standard deviations (SD) are indicated in Table 1. The solid line separates the non-European from the European observatories. Most of the averaged biases for all three components are lower than $\pm 200 \mathrm{nT}$. Generally, for $X$ and $Y$ components the observatories are characterized by low biases, and for the $Z$ component by larger biases. Three observatories have large biases in all components - LRV, PPT and SBA. The maximum bias in both $X$ and $Z$ appears at SBA ( -2204 and $-3748 \mathrm{nT}$, respectively), and in $Y$ at PPT $(-1039 \mathrm{nT})$. Let us note here that the European CM4-based biases averaged over 42 years (1960-2001) are also typically lower than $\pm 200 \mathrm{nT}$, and the largest values are again found in $Z$ (Verbanac et al., 2007a).

\subsection{Variations around the average observatory biases}

We further examine both the long-term trends and the remaining short-period variations around the average observatory biases, at all observatory locations. In Fig. 1 we show two examples of monthly bias time series at the NGK (Fig. 1, top) and KAK (Fig. 1, bottom) observatories. The average of each component bias is shown as a dashed line to emphasize the type of variations around the mean value. Note that the vertical scales are not the same, due to the large differences among averaged biases.
The simple oscillatory pattern around the average biases are observed in all bias time series, indicating that the crustal field has probably not changed over the considered time. This allows us to conclude that the computed average biases reflect the crustal field local contributions, $\boldsymbol{B}_{\mathrm{c}}$. Thus, the variations around the average observatory bias at an individual location can be equalized with $\epsilon$. In the following we focus on $\epsilon$ time series.

In all $\epsilon$ time series, we notice the trend related to the solar cycle. The observed trend reflects the general state of the heliosphere, namely the decrease of the interplanetary magnetic field (IMF) between 2000 and 2009. The decay of the IMF strength, especially over the late declining phase and the minimum of the solar cycle 23 (2006-2009), reduces the external field influences in the observatory biases seen as increasing or decreasing bias values.

Furthermore, for all components short-period $\epsilon$ variations are observed. They clearly show a semi-annual variation pattern, which we assign to the external field contributions. The other signals that contribute to $\epsilon$, as possible errors in the input data, are superimposed on the dominant external field signal. The amplitudes of semi-annual variations are related to the solar cycle phases, being remarkably larger from 2000 to 2005 than 2006-2009. During the first period they approximately amount for 30-75 $\mathrm{nT}$ in $X, 5-30 \mathrm{nT}$ in $Y$, and 10$75 \mathrm{nT}$ in $Z$. From 2006 to 2009, the amplitudes reach 10$40 \mathrm{nT}$ in $X, 0-20 \mathrm{nT}$ in $Y$, and 5-60 nT in $Z$.

The variation patterns at neighboring observatories are remarkably similar, although the averaged biases are often very different, indicating the homogeneous external field contributions within a pretty small region. In order to search for common patterns and find possible regional effects, we need to consider a region that is densely covered by geomagnetic observatories; we chose a region between 40 and $70^{\circ}$ geomagnetic latitude and between 90 and $120^{\circ}$ geomagnetic longitude. In the following we focus on the European observatories only (see the last 16 observatories listed in Table 1) and investigate in detail the temporal evolution of $\epsilon$. We display the monthly $\epsilon$ over the 9 years in form of a color-coded matrix for each component (Fig. 2). The observatory $\epsilon$ (lines of the matrices) are ordered by geomagnetic coordinates, with the most northern one at the top. To underline any pattern on short timescales, the color scale is limited to $20 \mathrm{nT}$, the stronger trends appearing as changes from dark blue to dark red or vice versa, without information about the real magnitude. Clear semi-annual variations can be noticed, with larger extremes that appear mostly around October and November, and around May and June of each year. These variations are especially prominent between 2000 and 2005, the period embracing the maximum and the very early declining phase of solar cycle 23. Let us note that the peak in fall 2000 and the spring peaks in 2003 and 2005 have lower values than other peaks. An extra peak appears in March 2003, also lower than the common ones. Over the period corresponding to the late 
Table 1. Average of the bias over 9 years (June 2000-August 2009) for $X, Y$ and $Z$ components at 42 observatories, in nanoteslas. The solid line separates the non-European from the European observatories.

\begin{tabular}{|c|c|c|c|c|c|c|c|c|c|}
\hline Code $^{a}$ & Long $^{\mathrm{b}}$ & $\mathrm{Lat}^{\mathrm{b}}$ & $h^{\mathrm{c}}$ & $X_{\text {avg }}$ & $\mathrm{SD} x$ & $Y_{\text {avg }}$ & SD $y$ & $Z_{\text {avg }}$ & $\mathrm{SD} z$ \\
\hline THL & 290.833 & 77.483 & 57 & -58.33 & 10.10 & 98.36 & 4.29 & 37.65 & 24.64 \\
\hline RES & 265.105 & 74.690 & 30 & 45.80 & 13.56 & 35.35 & 7.19 & 103.48 & 21.31 \\
\hline CBB & 254.969 & 69.123 & 20 & 69.45 & 17.59 & -114.45 & 13.99 & 102.25 & 18.08 \\
\hline BLC & 263.988 & 64.318 & 30 & 163.70 & 17.26 & -32.42 & 7.54 & -88.35 & 18.23 \\
\hline BRW & 203.380 & 71.320 & 12 & 17.90 & 19.81 & -70.71 & 8.22 & -23.61 & 18.24 \\
\hline YKC & 245.518 & 62.482 & 198 & -1.02 & 15.32 & -46.95 & 4.19 & -163.35 & 15.20 \\
\hline FCC & 265.912 & 58.759 & 15 & -121.58 & 13.95 & 63.28 & 3.29 & -252.20 & 13.49 \\
\hline $\mathrm{CMO}$ & 212.140 & 64.870 & 197 & 0.88 & 17.14 & -5.19 & 7.65 & -1.03 & 10.84 \\
\hline MEA & 246.653 & 54.616 & 700 & 92.31 & 13.96 & 15.67 & 3.72 & -133.67 & 8.18 \\
\hline STJ & 307.323 & 47.595 & 100 & 32.08 & 8.67 & 44.17 & 3.26 & 32.24 & 6.00 \\
\hline OTT & 284.448 & 45.403 & 75 & 122.46 & 10.14 & -133.92 & 1.58 & 149.54 & 7.24 \\
\hline VIC & 236.580 & 48.520 & 197 & 16.22 & 11.41 & 6.74 & 2.61 & -269.21 & 8.40 \\
\hline BOU & 254.767 & 40.140 & 1682 & -27.13 & 11.58 & 32.18 & 1.88 & -125.36 & 8.81 \\
\hline FRD & 282.633 & 38.210 & 69 & 56.86 & 10.09 & -51.35 & 1.41 & 137.90 & 7.47 \\
\hline NVS & 83.230 & 54.850 & 130 & 44.74 & 11.84 & -54.28 & 2.02 & 34.63 & 9.21 \\
\hline KAK & 140.186 & 36.232 & 36 & -14.80 & 13.01 & 10.07 & 1.56 & -86.22 & 4.54 \\
\hline $\mathrm{HON}$ & 202.000 & 21.320 & 4 & -185.19 & 12.97 & 80.80 & 3.10 & -320.61 & 3.91 \\
\hline MBO & 343.030 & 14.380 & 7 & 100.71 & 11.54 & 38.71 & 1.97 & 75.91 & 5.17 \\
\hline ABG & 72.872 & 18.638 & 7 & -99.42 & 18.81 & 434.06 & 7.18 & 713.92 & 10.75 \\
\hline GUA & 144.870 & 13.590 & 140 & 108.58 & 13.00 & 74.54 & 2.40 & 45.03 & 4.39 \\
\hline PPT & 210.426 & -17.567 & 357 & -922.02 & 15.29 & -1039.73 & 8.72 & -398.55 & 5.16 \\
\hline GNA & 115.947 & -31.780 & 60 & -35.50 & 11.73 & -100.83 & 2.56 & 96.53 & 7.35 \\
\hline CZT & 51.867 & -46.431 & 155 & -761.84 & 12.66 & 1092.13 & 3.77 & 147.66 & 6.08 \\
\hline PAF & 70.262 & -49.353 & 35 & 439.90 & 9.76 & -141.86 & 4.76 & -322.27 & 6.11 \\
\hline DRV & 140.007 & -66.667 & 30 & -155.60 & 13.68 & -420.01 & 4.18 & -2838.61 & 19.56 \\
\hline SBA & 166.763 & -77.850 & 16 & -2204.59 & 8.93 & -970.29 & 11.05 & -3748.76 & 33.56 \\
\hline LRV & 338.300 & 64.183 & 5 & -289.62 & 11.28 & 601.40 & 3.95 & -487.13 & 11.08 \\
\hline TRO & 18.948 & 69.663 & 105 & 30.75 & 13.14 & -393.09 & 4.04 & 146.89 & 16.94 \\
\hline $\mathrm{ABK}$ & 18.823 & 68.358 & 380 & 6.19 & 12.42 & 63.02 & 3.35 & 42.95 & 11.29 \\
\hline LER & 358.817 & 60.138 & 85 & -136.18 & 8.41 & 176.22 & 2.99 & 57.26 & 8.55 \\
\hline ESK & 356.794 & 55.314 & 245 & 1.09 & 8.21 & -32.52 & 3.27 & -35.81 & 10.71 \\
\hline VAL & 349.750 & 51.933 & 14 & 106.90 & 8.70 & -42.43 & 2.60 & -1.01 & 8.32 \\
\hline WNG & 9.073 & 53.743 & 50 & 43.76 & 9.38 & 46.63 & 2.54 & -53.22 & 8.86 \\
\hline HAD & 355.516 & 50.995 & 95 & -51.70 & 8.74 & 18.34 & 2.62 & 91.28 & 7.88 \\
\hline HLP & 18.816 & 54.608 & 1 & 37.56 & 11.23 & -163.66 & 2.85 & -85.99 & 8.59 \\
\hline NGK & 12.675 & 52.072 & 78 & -31.60 & 9.42 & 0.99 & 2.77 & -62.28 & 8.32 \\
\hline DOU & 4.599 & 50.100 & 225 & 5.37 & 9.70 & -15.50 & 2.78 & 99.60 & 8.27 \\
\hline BEL & 20.789 & 51.836 & 173 & 105.20 & 10.59 & 140.15 & 3.25 & 324.94 & 7.72 \\
\hline CLF & 2.270 & 48.020 & 145 & -82.09 & 9.67 & -13.00 & 2.90 & 124.78 & 7.48 \\
\hline HRB & 18.190 & 47.873 & 120 & 11.12 & 9.68 & -18.34 & 2.65 & -67.00 & 7.78 \\
\hline THY & 17.893 & 46.900 & 187 & -28.92 & 10.66 & -10.14 & 2.75 & -40.54 & 6.46 \\
\hline EBR & 0.333 & 40.957 & 531.5 & -16.76 & 12.30 & 3.82 & 5.70 & 15.721 & 7.44 \\
\hline
\end{tabular}

${ }^{a}$ According to the IAGA convention; ${ }^{b}$ observatory geographic coordinates in degrees; ${ }^{c}$ observatory altitudes in meters.

declining phase of solar cycle 23, from 2006 to 2009, the semi-annual variations are highly reduced.

\subsection{Comparison with MAGSAT and Ørsted biases}

As the computed average biases represent suitable estimations of the local crustal field over 9 years, we can compare them with MAGSAT and Ørsted based biases calculated by Mandea and Langlais (2002) for 39 observatories common to both studies. We aim to gain a better understanding of the possible crustal field changes over a longer time (3 decades). Since MAGSAT and Ørsted biases are given for epochs 1979.92 and 1999.92, respectively, we further compute average biases over the studied period by using only November and December values of each year. For most observatories, the averaged biases are in good agreement with 

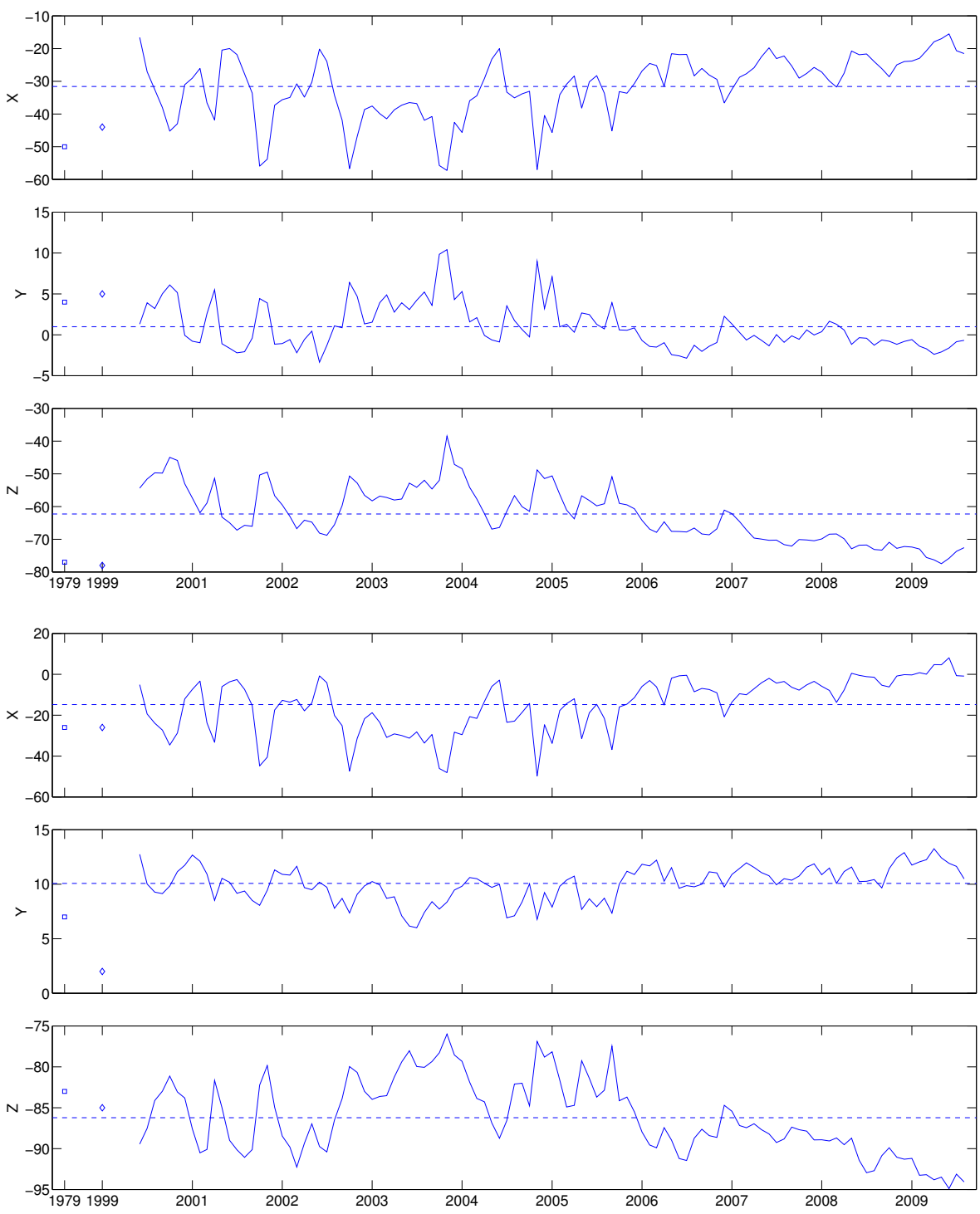

Figure 1. $X, Y$ and $Z$ monthly observatory biases over 9 years (June 2000-August 2009) at the NGK (top) and KAK (bottom) observatories, in nanoteslas. The average bias in each component is depicted by the dashed lines. The points at epochs 1979.92 and 1999.92 represent MAGSAT- and Ørsted-based biases computed by Mandea and Langlais (2002).

those based on MAGSAT and Ørsted data, the differences between them being within the range of the SD of averaged biases. At the NVS observatory, biases at 1979.92 and 1999.92 epochs differ significantly from our averaged biases. We have found that the larger disagreement found at PPT is due to a discontinuity in data in 2002.2 and, to correct that, we have applied the corrections of 221, 94, and $479 \mathrm{nT}$ in $X, Y$, and $Z$, respectively. Furthermore, we have noted that the biases at PPT are important because in the vicinity of the sensor there are large spatial gradients in the magnetic field. At some observatories the differences between biases are about $10 \mathrm{nT}$ larger than the SD of averaged bias values. Most of them are found in $Z$. Let us underline that for some of these observatories (e.g., ABG, FCC) MAGSAT and Ørsted biases, calculated by Mandea and Langlais (2002), differ from those published by other authors (e.g., Langel et al., 1982; Gubbins and Bloxham, 1985; Bloxham and Gubbins, 1986; Langel, 1987). Several possibilities can be invoked to explain these differences in biases: a different core field description by geomagnetic models, different methods and different observatory coordinates used in the previous studies, data errors or likely real changes in the crustal magnetization.

\section{Conclusions}

When using the observatory data for the core field modeling, it is crucial that the observatory biases are accurately determined and thereafter applied to the raw data. To study the ob- 

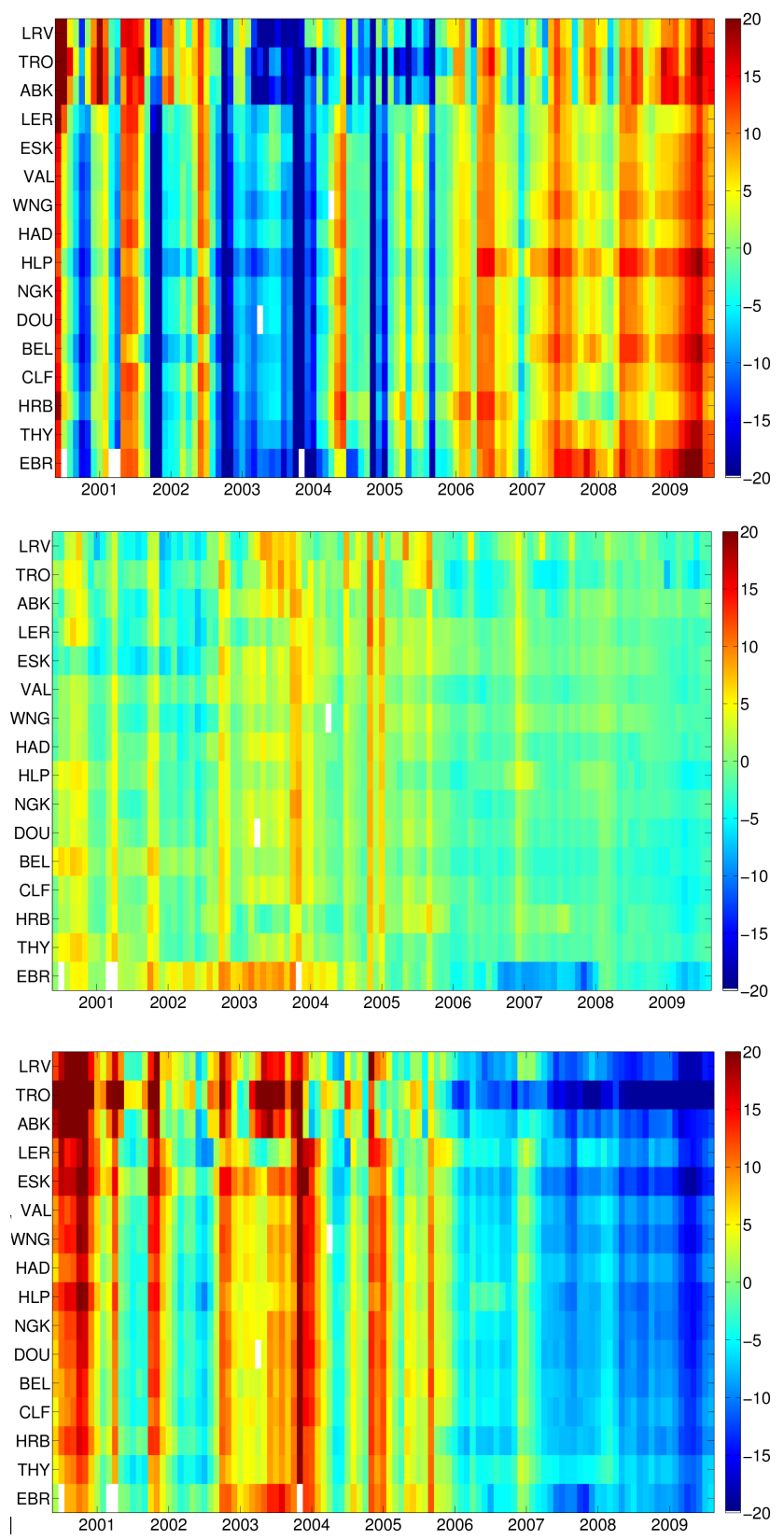

Figure 2. Variations around the $X, Y$ and $Z$ average monthly biases of the European observatories over 9 years, from June 2000 to August 2009, in nanoteslas. The observatories ( $y$ axes) are ordered by geomagnetic coordinates (the northernmost one at the top). The magnetic field components are expressed in geodetic coordinates.

servatory biases' temporal evolution over 9 years (from 2000 to 2009), we compute the monthly biases at 42 observatories by comparing the observatory data to the core field predictions given by the G model, entirely based on the CHAMP satellite data. We focus on both long-term trends and shortperiod variations in biases. For all components at each observatory we compute the average of biases over 9 years and analyze the variations around these averages. Finally, the com- parison with MAGSAT- and Ørsted-based biases computed for epochs 1979.92 and 1999.92 is performed.

The main findings of this study are as follows:

- the simple oscillatory pattern around the average observatory biases indicates that the crustal field has probably not changed over the considered time;

- the computed bias averages are a reliable estimation of $\boldsymbol{B}_{\mathrm{c}}$;

- the comparison of the average biases computed by using only November and December values of each year with both MAGSAT and Ørsted biases related to epochs 1979.92 and 1999.92, respectively, indicates that the crustal field at different locations has probably remained constant even over the last 3 decades;

- since the larger biases averaged over 9 years are mainly seen in the $Z$ component, and the differences between biases at various epochs are also observed in this component, it seems likely that they may be the signature of an induced magnetic field (a separate study dedicated to this issue is in progressing work);

- variations around the average observatory bias at individual locations can be estimated by $\boldsymbol{\epsilon}$;

- the long-trend seen in $\epsilon$ reflects the $\sim 11$ years of solar cycle influences, related to the general state of the heliosphere;

- the short-period variations observed in the $\epsilon$ time series are related to the external field (the amplitudes of these variations are in phase with different solar cycle periods, being remarkably larger over the period 2000-2005 than 2006-2009);

- clear semi-annual variations are observed in $\epsilon$ time series, with larger extremes appearing mostly around October and November, and around May and June of each year in $X$, and vice-versa in $Y$ and $Z$;

- a common external field pattern is found for European monthly biases; a dependence of the bias monthly variations on geomagnetic latitudes is not found for nonEuropean observatories.

Finally, let us note that the superposition of several signals with varying periods in each of the observatory bias components suggests that the involved processes which create complicated spatial and temporal behavior of biases are far from simple.

We plan to continue this study by investigating a better coverage with geomagnetic observatories over geomagnetic latitudes. A possible improvement would also be to quantify the different magnetic field contributions by applying methods presented in our previous studies (Verbanac et al., 2007a, 
b) but using monthly biases instead of annual mean biases. With the data from the new satellite mission Swarm and the above proposed extension of our study, we might be able to get a better insight into the temporal evolution of observatory biases, regarding both long-term trends and short period variations.

Acknowledgements. The results presented in this paper are based on data collected at magnetic observatories. We thank the observatory staff, INTERMAGNET and WDC Edinburgh for promoting high standards of magnetic observatory practice. The minute and hourly data of all geomagnetic components that we used are available at: http://www.intermagnet.org and http://www.wdc.bgs.ac.uk. We thank V. Lesur for providing us with $G$ model spherical harmonic coefficients, which we used to estimate the core field contribution at observatory locations. We gratefully acknowledge constructive suggestions from two anonymous reviewers.

Edited by: C. Gaina

\section{References}

Bloxham, J. and Gubbins, D.: Geomagnetic field analysis-IV. Testing the frozen-flux hypothesis, Geophys. J. R. Astr. Soc., 84, 139-152, doi:10.1111/j.1365-246X.1986.tb04349.x, 1986.

Campbell, W. H.: Earth Magnetism - A Guided Tour through Magnetic Fields, Harcourt/Academic Press, San Diego, 22-23, 2001

Gubbins, D. and Bloxham, J.: Geomagnetic field ananlysisIII. Magnetic fields on the core-mantle boundary, Geophys. J. R. Astr. Soc., 80, 695-713, doi:10.1111/j.1365246X.1985.tb05119.x, 1985.

Jacobs, J. A.: Geomagnetism, Vol. 2, Academic Press, London, Orlando, 579 pp., 1987.
Langel, R. A.: The Main Field, in: Geomagnetism, edited by: Jacobs, J. A., Academic Press, London, 1, 249-512, 1987.

Langel, R. A. and Hinze, W. J.: The magnetic field of the Earth's lithosphere: The satellite perspective, Cambridge Univ. Press, Cambridge, 429 pp., 1998.

Langel, R. A., Estes, R. H., and Mead, G. D.: Some new methods in geomagnetic field modeling applied to the 1960-1980 epoch, J. Geomag. Geoelectr., 34, 327-349, 1982.

Lesur, V. and Gubbins, D.: Using geomagnetic secular variation to separate remanent and induced sources of the crustal magnetic field, Geophys. J. Int., 142, 889-897, 2000.

Lesur, V., Whaler, K., and Wardinski, I.: Are geomagnetic data consistent with stably stratified flow at the core-mantle boundary?, Geophys. J. Int., 201, 929-946, doi:10.1093/gji/ggv031, 2015.

Mandea, M. and Langlais, B.: Observatory crustal magnetic biases during MAGSAT and Oersted satellite missions, Geophys. Res. Lett., 29, 11-15, 2002.

Mandea, M. and Thèbault, E.: The Changing Faces of the Earth's Magnetic Field: a Glance at the Magnetic Lithospheric Field, from Local and Regional Scales to a Planetary View, Commission for the Geological Map of the World, Paris, 49 pp., ISBN:978-2-9517181-9-7, 2007.

Merrill, R., McElhinny, M., and McFadden, P.: The Magnetic Field of the Earth: Paleomagnetism, the Core, and the Deep Mantle, Academic Press, San Diego, 305-377, 1998.

Sabaka, T., Olsen., N., and Langel, R.: A comprehensive model of the quiet-time, near Earth magnetic field:phase3, Geophys. J. Int., 151, 32-68, 2002.

Verbanac, G., Korte, M., and Mandea, M.: On long-term trends of the European geomagnetic observatory biases, Earth Planets Space, 59, 685-695, 2007a.

Verbanac, G., Lühr, H., Rother, M., Korte, M., and Mandea, M.: Contribution of the external field to the observatory annual means and a proposal for their corrections, Earth Planets Space, 59, 251-257, 2007b. 